\title{
Counting rational points on cubic and quartic surfaces
}

\author{
by
}

\author{
T. D. Browning (Oxford)
}

1. Introduction. Let $V$ be a non-singular cubic surface defined over $\mathbb{Q}$, and let $U$ be the open subset obtained by deleting the 27 lines; for given $B \geq 1$, we shall be concerned with the number $\mathcal{N}_{U}(B)$ of rational points in $U$ of height at most $B$. The behaviour of $\mathcal{N}_{U}(B)$ is predicted as a special case of Manin's conjecture [4], which states that if $r$ is the rank of the Néron-Severi group of $V$ then the asymptotic behaviour

$$
\mathcal{N}_{U}(B)=c_{V} B(\log B)^{r-1}(1+o(1))
$$

should hold for some constant $c_{V}>0$, whenever $U(\mathbb{Q})$ is non-empty. Although Slater and Swinnerton-Dyer [13] have established a lower bound with the correct order of magnitude, whenever the surface contains two rational skew lines, no non-singular cubic surface is known for which the corresponding upper bound can be proved. We shall therefore be concerned with the somewhat weaker conjecture that

$$
\mathcal{N}_{U}(B)=O_{\varepsilon, V}\left(B^{1+\varepsilon}\right),
$$

where the implied constant may depend upon the choice of $\varepsilon>0$ and $V$.

Aside from the surface $x_{1}^{3}+x_{2}^{3}+x_{3}^{3}+x_{4}^{3}=0$-for which Hooley [9] proves the upper bound $O_{\varepsilon}\left(B^{5 / 3+\varepsilon}\right)$ by means of sieve methods - one of the earliest attempts at estimating $\mathcal{N}_{U}(B)$ in general is due to Manin and Tschinkel [11]. They establish the bound $O_{\varepsilon, V}\left(B^{5 / 3+\varepsilon}\right)$ for any non-singular cubic surface $V$, in which all of the lines are rational. Inspired by the particular surface studied by Hooley, Heath-Brown [6] considers the case of arbitrary non-singular cubic surfaces containing three rational coplanar lines, and ultimately achieves a bound with exponent $4 / 3+\varepsilon$ for such surfaces. In a later paper he conditionally extends this bound to all non-singular cubic surfaces [7], subject to the hypothesis that the estimate

$$
r_{E}=o\left(\log C_{E}\right) \quad \text { as } r_{E} \rightarrow \infty
$$

2000 Mathematics Subject Classification: Primary 11D45; Secondary 11G35, 14 G05. 
should hold uniformly for any elliptic curve $E$ of rank $r_{E}$ and conductor $C_{E}$, and which in itself follows from standard hypotheses concerning the $L$-function of an elliptic curve. To date, the best unconditional upper bound for the number of non-trivial points on a cubic surface is $O_{\varepsilon}\left(B^{52 / 27+\varepsilon}\right)$, again due to Heath-Brown [8, Theorem 6]. In particular it shows that the contribution of at least $B^{2}$ from any rational lines in $V$ will always dominate $\mathcal{N}_{U}(B)$. Furthermore, it has the advantage of an implied constant which is completely independent of the individual surface. Our principal result arises from relaxing the constraints placed by Heath-Brown upon the existence of three rational coplanar lines in his earlier work, and may be viewed as a modest step on the way towards a better unconditional bound for general non-singular cubic surfaces.

TheOREM 1. Let $V$ be a non-singular cubic surface over $\mathbb{Q}$. If $V$ contains a rational line, then for any $\varepsilon>0$ we have

$$
\mathcal{N}_{U}(B)=O_{\varepsilon, V}\left(B^{46 / 25+\varepsilon}\right) .
$$

The basic philosophy behind the proof of Theorem 1 will be to translate the problem either to a family of curves of degree two (via an obvious morphism $\varrho: V \rightarrow \mathbb{P}^{1}$ ), or to a smaller family of surfaces of degree four (via an alternative morphism $\phi: V \rightarrow \mathbb{P}^{4}$ ), according to the size of a certain highest common factor. In fact the technique can be easily applied to non-singular surfaces of higher degree, provided that they are of a suitable shape. As an example of this we examine the case of non-singular quartic surfaces $W \subset \mathbb{P}^{3}$ defined over $\mathbb{Q}$, and set $U$ to be the open subset obtained by deleting all of the lines from $W$. Unlike in the case of cubic surfaces, there seems to be no conjectural understanding of how the quantity $\mathcal{N}_{U}(B)$ should behave for given $B \geq 1$, although it seems sensible to strive for a majoration of the form (1) for arbitrary irreducible quartic surfaces $W$.

The best available upper bound for the number of non-trivial points on a non-singular quartic surface is currently $O_{\varepsilon}\left(B^{16 / 9+\varepsilon}\right)$, due to Heath-Brown $[8$, Theorem 10]. As with the previously mentioned bound for cubic surfaces, the implied constant is independent of the individual surface. This has been improved upon only in a few specific cases. Most notably, Hooley [10] has provided the upper bound $O_{\varepsilon}\left(B^{5 / 3+\varepsilon}\right)$ for the surface $x_{1}^{4}+x_{2}^{4}=x_{3}^{4}+x_{4}^{4}$, by means of very delicate sieve methods. This has been conditionally improved to $O_{\varepsilon}\left(B^{8 / 5+\varepsilon}\right)$ by Browning [2], subject to the hypothesis (2) above. We turn in Section 4 to the proof of the following result, which conditionally improves upon Heath-Brown's result whenever the surface contains two coplanar rational lines.

TheOREM 2. Let $W$ be a non-singular quartic surface over $\mathbb{Q}$. If $W$ contains two coplanar rational lines, then for any $\varepsilon>0$ we have 


$$
\mathcal{N}_{U}(B)=O_{\varepsilon, W}\left(B^{26 / 15+\varepsilon}\right),
$$

provided that the rank hypothesis (2) holds.

In the course of this work, the author has benefited from many useful conversations with Jean-Louis Colliot-Thélène and Roger Heath-Brown. A number of helpful comments were made by the referee, whose aid is also gratefully acknowledged.

2. Preliminaries. The first and most versatile result is an elementary slicing result due to Siegel (see Skinner and Wooley [12, Lemma 2.1], for example). The proof is an easy application of the pigeon-hole principle.

LEMMA 1. For $n \geq 2$ and arbitrary real $B_{1}, \ldots, B_{n} \geq 1$, let $\mathbf{x} \in \mathbb{Z}^{n}$ lie in the box $\left|x_{i}\right| \leq B_{i}$ for $1 \leq i \leq n$. Then there exists a primitive non-zero vector $\mathbf{y} \in \mathbb{Z}^{n}$ for which $\mathbf{x} . \mathbf{y}=0$ and $\left|y_{i}\right| \leq B_{i}^{-1}\left(n B_{1} \ldots B_{n}\right)^{1 /(n-1)}$ for $1 \leq i \leq n$.

The remaining results concern the distribution of rational points on general hypersurfaces, and as such we first need to introduce some notation. Given $n \geq 2$ and a non-zero form $f\left(x_{1}, \ldots, x_{n}\right)$ of degree $d \geq 1$ with rational coefficients, we define the quantity

$$
\mathcal{N}(f ; \mathbf{B}):=\#\left\{\mathbf{x} \in \mathbb{Z}^{n}: \mathbf{x} \text { primitive, }\left|x_{i}\right| \leq B_{i}(1 \leq i \leq n), f(\mathbf{x})=0\right\}
$$

for given $B_{1}, \ldots, B_{n} \geq 1$. Here, an integer vector $\mathbf{x}$ is said to be primitive if there is no common non-trivial divisor of the coefficients $x_{i}$. We write $\mathcal{N}(f ; B)$ in the case $B=B_{i}$ for $1 \leq i \leq n$. One may easily modify this counting function in order to ignore points lying on certain subvarieties of $f=0$, such as the focus of this paper $\mathcal{N}_{U}(B)$ for example. Throughout this section, the implied constant in each estimate may depend on $d$ and $n$; any further dependence will be explicitly indicated. We begin by giving a rather crude upper bound for $\mathcal{N}(f ; \mathbf{B})$, though which uniformly applies to arbitrary forms $f$ of degree $d \geq 1$, and is best possible in the case of linear forms. The proof is an easy induction argument on the number of variables $n$, precisely as given by Heath-Brown [8, Theorem 1] in the case $B=B_{i}$ for example.

LEMMA 2. For $n \geq 2$ and given $B_{1}, \ldots, B_{n} \geq 1$, we have

$$
\mathcal{N}(f ; \mathbf{B}) \ll \frac{B_{1} \ldots B_{n}}{\min _{1 \leq i \leq n} B_{i}} .
$$

Shifting to available estimates for $\mathcal{N}(f ; \mathbf{B})$ in the case $n=4$, HeathBrown $\left[8\right.$, Theorem 9] has provided the sharper bound $\mathcal{N}(f ; B)=O_{\varepsilon}\left(B^{2+\varepsilon}\right)$ whenever $d \geq 2$ and the form $f$ is irreducible. Here, as in the remainder of this paper, irreducibility is taken to mean irreducibility over $\mathbb{C}$. Browning [3, Theorem 13] achieves the following version for points lying in a general box, with very minor modifications to Heath-Brown's original arguments. 
Lemma 3. For $n=4$ and given $1 \leq B_{1} \leq B_{2} \leq B_{3} \leq B_{4}$, we have

$$
\mathcal{N}(f ; \mathbf{B}) \ll_{\varepsilon} B_{3} B_{4}^{1+\varepsilon}
$$

whenever $f$ is an irreducible form of degree at least 2 .

It is easy to see that this is essentially best possible, given that the line $x_{1}=x_{2}=0$ may be contained in the surface.

Finally we consider the case $n=3$ of plane curves, and fix the notation

$$
V=B_{1} B_{2} B_{3}, \quad T=\max \left\{B_{1}^{f_{1}} B_{2}^{f_{2}} B_{3}^{f_{3}}\right\},
$$

where the maximum is taken over all integer triples $\left(f_{1}, f_{2}, f_{3}\right)$ for which the corresponding monomial $x_{1}^{f_{1}} x_{2}^{f_{2}} x_{3}^{f_{3}}$ has a non-zero coefficient in $f$. Then Heath-Brown [8, Theorem 3] has provided the following versatile result.

Lemma 4. Let $\varepsilon>0$. If $f$ is an irreducible ternary form of degree $d \geq 2$, then

$$
\mathcal{N}(f ; \mathbf{B}) \ll_{\varepsilon} T^{-d^{-2}} V^{d^{-1}+\varepsilon} .
$$

When $f$ is non-singular, Heath-Brown establishes the lower bound $T \geq$ $V^{d / 3}$, which obviously implies

$$
\mathcal{N}(f ; \mathbf{B}) \ll_{\varepsilon} V^{2 /(3 d)+\varepsilon} .
$$

Whenever $f$ is irreducible but possibly singular, this lower bound is no longer uniformly possible. It is clear that if $B_{1} \leq B_{2} \leq B_{3}$ say, then the bound $T \geq B_{1}^{d}$ is trivial. However we observe that for irreducible $f$, there must be some monomial appearing in $f$ with non-zero coefficient which does not contain $x_{1}$ as a factor. But then $T \geq B_{2}^{d}$, so that for irreducible $f$ we obtain

$$
\mathcal{N}(f ; \mathbf{B}) \ll_{\varepsilon}\left(B_{1} B_{3}\right)^{1 / d+\varepsilon} .
$$

Lemma 4 can also be used to retrieve a bound due to Bombieri and Pila [1] for the number of integral points lying in a box of side $2 B$ on affine plane curves. Indeed, we may consider a projective model $f\left(x_{1}, x_{2}, x_{3}\right)$ of any given irreducible polynomial $g(x, y)$ of degree $d \geq 2$, and so deduce that

(6) $\#\left\{(x, y) \in \mathbb{Z}^{2}:|x|,|y| \leq B, g(x, y)=0\right\} \leq \mathcal{N}(f ; B, B, 1) \ll_{\varepsilon} B^{1 / d+\varepsilon}$,

since then $T=B^{d}$. Clearly the estimate holds trivially in the case of linear polynomials $g(x, y)$.

In the special case $d=2$, we shall need in Section 3.2 a version of (4) which takes into account the size of the coefficients of the form [8, Corollary 2].

LEMma 5. Let $q$ be a non-singular ternary quadratic form over $\mathbb{Z}$ with matrix $\mathbf{M}$. Let $\Delta_{q}=|\operatorname{det} \mathbf{M}|$, and let $D_{q}$ be the highest common factor of the $2 \times 2$ minors of $\mathbf{M}$. Then for given $B_{1}, B_{2}, B_{3} \geq 1$, we have

$$
\mathcal{N}(q ; \mathbf{B}) \ll_{\varepsilon}\left\{1+\left(\frac{B_{1} B_{2} B_{3} D_{q}^{3 / 2}}{\Delta_{q}}\right)^{1 / 3+\varepsilon}\right\} \Delta_{q}^{\varepsilon} .
$$


Whenever $d=3$ and $f$ is non-singular, so that $f=0$ defines an elliptic curve whenever the set of rational points $f(\mathbb{Q})$ is non-empty, we shall need the following conditional result in Section 4.2. It is due to Heath-Brown [7, Theorem 3], and may be considered as a uniform version of Néron's classical asymptotic formula $\mathcal{N}(f ; B) \sim c_{f}(\log B)^{r_{f} / 2}$, in terms of the curve's rank $r_{f}$. We use the notation $\|f\|$ to denote the maximum modulus of the coefficients of $f$.

Lemma 6. Let $E=0$ be the equation of an elliptic curve defined over $\mathbb{Q}$, and let $\varepsilon>0$. Then if the rank hypothesis (2) holds, we have

$$
\mathcal{N}(E ; B) \ll_{\varepsilon} B^{\varepsilon}\|E\|^{\varepsilon} .
$$

3. Non-singular cubic surfaces. Let $B \geq 1$, and let $F \in \mathbb{Z}\left[x_{1}, x_{2}\right.$, $\left.x_{3}, x_{4}\right]$ be the non-singular cubic form defining $V$. Then $\mathcal{N}_{U}(B)$ is the total number of primitive $\mathbf{x}=\left(x_{1}, x_{2}, x_{3}, x_{4}\right) \in \mathbb{Z}^{4}$ lying in the box $\left|x_{i}\right| \leq B$ $(1 \leq i \leq 4)$, such that $F(\mathbf{x})=0$, but $\mathbf{x}$ does not lie on any line contained the surface. It is clear that for the purposes of proving Theorem 1, it suffices to replace $F$ by any form equivalent to it over the rational numbers. The rational line which lies in the surface may be taken to be $x_{1}=x_{2}=0$, so that $F$ takes the form

$$
F(\mathbf{x})=x_{1} Q_{1}(\mathbf{x})-x_{2} Q_{2}(\mathbf{x})
$$

where $Q_{1}$ and $Q_{2}$ are integral quadratic forms. We shall use the special shape that $F$ takes, in order to take out the highest common factor of $x_{1}$ and $x_{2}$ in any integer zero $\mathbf{x}$ of $F$. Thus, for each such vector $\mathbf{x}$ we define $\lambda=\left(x_{1}, x_{2}\right)$, and

$$
\mathbf{w}=\left(x_{1} / \lambda, x_{2} / \lambda, x_{3}, x_{4}\right) .
$$

In particular $w_{1}$ and $w_{2}$ are coprime, and $\mathbf{w}$ lies in the range

$$
\left|w_{1}\right|,\left|w_{2}\right| \leq B / \lambda, \quad\left|w_{3}\right|,\left|w_{4}\right| \leq B
$$

For each $1 \leq \lambda \leq B$, we are now concerned with the number $\mathcal{M}_{U}(B ; \lambda)$ of primitive $\mathbf{w} \in \mathbb{Z}^{4}$ lying in this region for which $F_{\lambda}(\mathbf{w})=0$, where

$$
F_{\lambda}(\mathbf{w})=w_{1} Q_{1}\left(\lambda w_{1}, \lambda w_{2}, w_{3}, w_{4}\right)-w_{2} Q_{2}\left(\lambda w_{1}, \lambda w_{2}, w_{3}, w_{4}\right),
$$

and $\mathbf{w}$ does not lie on any line contained in the surface. In order to estimate the quantity $\mathcal{M}_{U}(B ; \lambda)$, we split our considerations according to the size of the variables $w_{1}, w_{2}$. Thus let $\mathcal{L}_{1}(B ; \lambda)$ denote the number of vectors $\mathbf{w}$ counted by $\mathcal{M}_{U}(B ; \lambda)$, for which

$$
\left|w_{1} w_{2}\right|>B^{46 / 25}
$$

and let $\mathcal{L}_{2}(B ; \lambda)$ denote the corresponding number of $\mathbf{w}$ for which

$$
\left|w_{1} w_{2}\right| \leq B^{46 / 25}
$$


It follows that

$$
\mathcal{N}_{U}(B) \leq \sum_{1 \leq \lambda<B^{2 / 25}} \mathcal{L}_{1}(B ; \lambda)+\sum_{1 \leq \lambda \leq B} \mathcal{L}_{2}(B ; \lambda)
$$

since $\mathcal{M}_{U}(B ; \lambda)=\mathcal{L}_{1}(B ; \lambda)+\mathcal{L}_{2}(B ; \lambda)$ and clearly only those $\lambda$ in the range $1 \leq \lambda<B^{2 / 25}$ lead to a non-zero value of $\mathcal{L}_{1}(B ; \lambda)$. The proof of Theorem 1 will follow from Sections 3.1 and 3.2 below, which will be concerned with estimating $\sum_{\lambda} \mathcal{L}_{1}(B ; \lambda)$ and $\sum_{\lambda} \mathcal{L}_{2}(B ; \lambda)$ respectively.

3.1. Estimating $\sum_{\lambda} \mathcal{L}_{1}(B ; \lambda)$. For ease of notation we first absorb each appearance of $\lambda$ into the coefficients of $Q_{1}$ and $Q_{2}$ in (10), and let $V_{\lambda}$ denote the surface $F_{\lambda}=0$. We note that there can be no point $\left[0,0, \eta_{3}, \eta_{4}\right] \in \mathbb{P}^{3}$ for which

$$
Q_{1}\left(0,0, \eta_{3}, \eta_{4}\right)=Q_{2}\left(0,0, \eta_{3}, \eta_{4}\right)=0
$$

holds, since it would produce a singularity in the surface $V_{\lambda}$. In particular the monomial $w_{3}^{2}$ must appear with non-zero coefficient in either $Q_{1}$ or $Q_{2}$, and we henceforth suppose without loss of generality that

$$
Q_{1}(0,0,1,0) \neq 0 \text {. }
$$

Our primary task in this section will be to estimate the number of primitive $\mathbf{w} \in \mathbb{Z}^{4}$ such that $F_{\lambda}(\mathbf{w})=0$, $\mathbf{w}$ does not lie on any line in the surface, and for which

$$
C_{1} / 2<\left|w_{1}\right| \leq C_{1}, \quad C_{2} / 2<\left|w_{2}\right| \leq C_{2}, \quad\left|w_{3}\right|,\left|w_{4}\right| \leq B,
$$

where

$$
B^{46 / 25} \ll C_{1} C_{2}, \quad 1 \leq C_{1}, C_{2} \leq B / \lambda .
$$

We shall ultimately sum our bounds for this quantity over powers of 2 , in order to get an estimate for $w_{1}, w_{2}$ lying in the general range defined by (9) and (11) for each $1 \leq \lambda<B^{2 / 25}$, and so an estimate for $\mathcal{L}_{1}(B ; \lambda)$.

We begin with a certain observation concerning the integer zeros of $F_{\lambda}(\mathbf{w})$, which is fundamental to Section 3.1 .2 below. Since $w_{1}$ and $w_{2}$ are coprime, it follows that there exists $w_{5} \in \mathbb{Z}$ such that

$$
Q_{1}\left(w_{1}, w_{2}, w_{3}, w_{4}\right)=w_{2} w_{5}, \quad Q_{2}\left(w_{1}, w_{2}, w_{3}, w_{4}\right)=w_{1} w_{5},
$$

where $Q_{1}$ and $Q_{2}$ are such that (14) holds. We denote this intersection of quadric hypersurfaces in $\mathbb{P}^{4}$ by $T_{\lambda}$, and observe that

$$
w_{5} \ll \frac{B^{2}}{\sqrt{C_{1} C_{2}}}
$$

since $C_{1}, C_{2} \ll B$. This rather ad-hoc arrangement in fact corresponds to the quadratic map $\phi: \mathbb{P}^{3} \rightarrow \mathbb{P}^{4}$ given by

$$
\phi\left[w_{1}, w_{2}, w_{3}, w_{4}\right]= \begin{cases}{\left[w_{1}^{2}, w_{1} w_{2}, w_{1} w_{3}, w_{1} w_{4}, Q_{2}\right],} & \left(w_{1}, Q_{2}\right) \neq(0,0), \\ {\left[w_{1} w_{2}, w_{2}^{2}, w_{2} w_{3}, w_{2} w_{4}, Q_{1}\right],} & \left(w_{2}, Q_{1}\right) \neq(0,0) .\end{cases}
$$


It is clear that the two alternatives are consistent when they both apply, and that $\phi$ is undefined only at the singular points $\left[0,0, \eta_{3}, \eta_{4}\right]$ of $V_{\lambda}$, by (13). Therefore $\phi: V_{\lambda} \rightarrow \mathbb{P}^{4}$ is a regular map with image $T_{\lambda}$. In fact it induces an isomorphism between the open sets $V_{\lambda}-L$ and $T_{\lambda}-\left\{p_{0}\right\}$, where $L$ is the line $w_{1}=w_{2}=0$ and $p_{0}=[0,0,0,0,1]$. We have used the coprimality of $w_{1}$ and $w_{2}$ in any integer solution counted by $\mathcal{L}_{1}(B ; \lambda)$, in order to deduce that $Q_{2} / w_{1}=Q_{1} / w_{2} \in \mathbb{Z}$; this thereby avoids the effect that $\phi$ has upon the height of the rational points in $V_{\lambda}-L$.

Returning to the cubic surface $V_{\lambda}$, Lemma 1 implies that for each integer $\mathbf{w}$ lying in the range (15), there exists a non-zero primitive $\mathbf{y} \in \mathbb{Z}^{4}$ such that $\mathbf{w} \cdot \mathbf{y}=0$, and

$$
y_{i} \ll \begin{cases}C_{i}^{-1}\left(C_{1} C_{2} B^{2}\right)^{1 / 3}, & i=1,2, \\ B^{-1}\left(C_{1} C_{2} B^{2}\right)^{1 / 3}, & i=3,4 .\end{cases}
$$

Furthermore, it is plain from (16) that each of the bounds on the right hand side of (19) is $\gg 1$. For each $\mathbf{y}$ lying in this range, it suffices to estimate the number of primitive integer $\mathbf{w}$ in the range (15) which lie on the curve

$$
C_{\mathbf{y}}: \quad \mathbf{w} \cdot \mathbf{y}=F_{\lambda}(\mathbf{w})=0,
$$

but not on any linear component of it. Whereas we handle the contribution from the singular $C_{\mathbf{y}}$ in a rather straightforward fashion, our key saving over previous approaches comes from the treatment of the non-singular $C_{\mathbf{y}}$, for which we translate our attention instead to the corresponding hyperplane section of the surface $T_{\lambda} \subset \mathbb{P}^{4}$.

We henceforth decompose all of the primitive $\mathbf{y} \in \mathbb{Z}^{4}$ lying in the range (19) into three disjoint sets $\mathfrak{Y}_{1} \cup \mathfrak{Y}_{2} \cup \mathfrak{Y}_{3}$. First, let $\mathfrak{Y}_{1}$ denote the set of all such $\mathbf{y}$ for which $C_{\mathbf{y}}$ is singular, so that in particular every $\mathbf{y} \in \mathfrak{Y}_{2} \cup \mathfrak{Y}_{3}$ leads to a non-singular curve $C_{\mathbf{y}}$. We set $\mathfrak{Y}_{2}$ to be the subset of such $\mathbf{y}$ for which the form in $y_{3}, y_{4}$

$$
y_{4} Q_{1}\left(0,0, y_{4},-y_{3}\right)
$$

vanishes. Finally, we let $\mathfrak{Y}_{3}$ be the remaining set of integer $\mathbf{y}$ lying in the range (19). It follows from (14) that the form (21) is not identically zero as a function of $y_{3}, y_{4}$, and so Lemma 2 implies that the set $\mathfrak{Y}_{2}$ contains at most $O(B)$ vectors $\mathbf{y}$.

3.1.1. Contribution from the case $\mathbf{y} \in \mathfrak{Y}_{1} \cup \mathfrak{Y}_{2}$. For each $\mathbf{y} \in \mathfrak{Y}_{1} \cup \mathfrak{Y}_{2}$ we turn our attention to the integer points $\mathbf{w}$ lying on the curve $C_{\mathbf{y}}$ for which $\mathbf{w}$ lies in the region (15). In order to get a handle on the set of vectors $\mathfrak{Y}_{1}$, we apply the theory of the dual surface. The dual equation $\widehat{F}_{\lambda}(\mathbf{u})=0$ is characterised by the existence of some $\mathbf{z} \in \mathbb{C}^{4}$ such that $\nabla F_{\lambda}(\mathbf{z})=\mathbf{u}$ and $F_{\lambda}(\mathbf{z})=0$. In particular, it is not hard to see that the plane section $C_{\mathbf{y}}$ is singular if and only if $\mathbf{y}$ satisfies the equation $\widehat{F}_{\lambda}(\mathbf{y})=0$; furthermore it is 
part of the general theory that $\widehat{F}_{\lambda}$ is an irreducible form of bounded degree at least 2, which we may take to be defined over $\mathbb{Z}$ (see [5, Example 15.22], for example). Lemma 3 therefore leads us to conclude that there are at most

$$
O_{\varepsilon}\left(\frac{B^{4 / 3+\varepsilon}}{\left(C_{1} C_{2}\right)^{1 / 3}}\right)
$$

vectors $\mathbf{y}$ lying in the range (19) for which $C_{\mathbf{y}}$ is singular.

Handling the case of singular $C_{\mathbf{y}}$ first, we suppose that $y_{4} \neq 0$ say. Then we may apply the bounds of Lemma 4 to the cubic form

$$
g_{\mathbf{y}}\left(w_{1}, w_{2}, w_{3}\right)=F_{\lambda}\left(y_{4} w_{1}, y_{4} w_{2}, y_{4} w_{3},-y_{1} w_{1}-y_{2} w_{2}-y_{3} w_{3}\right) .
$$

If $g_{\mathbf{y}}$ is irreducible we get a contribution $O_{\varepsilon}\left(\left(C_{1} C_{2}\right)^{1 / 6} B^{1 / 3+\varepsilon}\right)$, by (5). Moreover any linear factor of $g_{\mathbf{y}}$ will correspond to a line lying in the surface $F_{\lambda}=0$, and so will contribute nothing, while for a (necessarily non-singular) quadratic factor we have $O_{\varepsilon}\left(\left(C_{1} C_{2} B\right)^{1 / 3+\varepsilon}\right)$ points. Since $\left(C_{1} C_{2}\right)^{1 / 6} B^{1 / 3} \leq\left(C_{1} C_{2} B\right)^{1 / 3}$, it suffices to sum $O_{\varepsilon}\left(\left(C_{1} C_{2} B\right)^{1 / 3+\varepsilon}\right)$ over all of the vectors $\mathbf{y} \in \mathfrak{Y}_{1}$. Applying the bound (22) for the number of such $\mathbf{y}$, we therefore obtain the total contribution

$$
\ll_{\varepsilon} B^{5 / 3+\varepsilon}
$$

to $\mathcal{L}_{1}(B ; \lambda)$, from those $\mathbf{y} \in \mathfrak{Y}_{1}$ for which $y_{4} \neq 0$. It is clear that we obtain precisely the same bound arising from those $\mathbf{y} \in \mathfrak{Y}_{1}$ for which $y_{3} \neq 0$, and so it remains to consider the contribution from the non-zero $\left(y_{1}, y_{2}, 0,0\right) \in \mathfrak{Y}_{1}$ which give rise to a non-singular quadratic factor of $g_{\mathbf{y}}$. In fact we may as well assume that $y_{1} y_{2} \neq 0$ in each such $\mathbf{y}$, since the alternative contributes just $O_{\varepsilon}\left(B^{1+\varepsilon}\right)$ to $\mathcal{L}_{1}(B ; \lambda)$ by $(15),(4)$ and the fact that $\mathbf{y}$ is primitive. But then we may employ the bound (22) for the number of such $\left(y_{1}, y_{2}, 0,0\right) \in \mathfrak{Y}_{1}$, each of which corresponds to a contribution of at most

$$
\ll_{\varepsilon}\left(C_{1} C_{2}\right)^{1 / 6} B^{2 / 3+\varepsilon},
$$

since we are free to eliminate $w_{1}$ or $w_{2}$ in (20), according to which of $C_{1}$ or $C_{2}$ is the largest. This therefore yields the contribution

$$
\ll_{\varepsilon} \frac{B^{2+\varepsilon}}{\left(C_{1} C_{2}\right)^{1 / 6}} \ll B^{127 / 75+\varepsilon}
$$

by (16), which plainly exceeds $(23)$.

Turning to the contribution from the $\mathbf{y} \in \mathfrak{Y}_{2}$ - for which each $C_{\mathbf{y}}$ is nonsingular - we observe that $\mathbf{w}$ is contained in the cube of side $2 B$, so that we may apply Lemma 4 in the case $B_{i}=B$ for $1 \leq i \leq 3$. Upon eliminating any of the variables from (20), it suffices to sum the contribution $O_{\varepsilon}\left(B^{2 / 3+\varepsilon}\right)$ over every $\mathbf{y} \in \mathfrak{Y}_{2}$. Applying the bound $O(B)$ for the number of such $\mathbf{y}$, we therefore obtain a total contribution of $O_{\varepsilon}\left(B^{5 / 3+\varepsilon}\right)$ to $\mathcal{L}_{1}(B ; \lambda)$, which is bounded by (24). 
3.1.2. Contribution from the case $\mathbf{y} \in \mathfrak{Y}_{3}$. For each $\mathbf{y} \in \mathfrak{Y}_{3}$, the number of primitive integer vectors lying on the curve $C_{\mathbf{y}}$ in the region (15) is majorised by the number of primitive

$$
\mathbf{w}=\left(w_{1}, w_{2}, w_{3}, w_{4}, w_{5}\right) \in \mathbb{Z}^{5}
$$

in the region defined by (15) and (17) which lie on the curve $S_{\mathbf{y}}=H_{\mathbf{y}} \cap T_{\lambda}$ in $\mathbb{P}^{4}$; here $\mathbf{y}=\left(y_{1}, y_{2}, y_{3}, y_{4}, 0\right)$, and $H_{\mathbf{y}} \subset \mathbb{P}^{4}$ denotes the hyperplane $\mathbf{w} \cdot \mathbf{y}=0$. Since $y_{4} \neq 0$ for all $\mathbf{y} \in \mathfrak{Y}_{3}$, we may eliminate $w_{4}$ from the equations defining $S_{\mathbf{y}}$ in order to obtain the explicit model

$$
S_{\mathbf{y}}:\left\{\begin{array}{l}
R_{1}\left(w_{1}, w_{2}, w_{3}\right)-y_{4}^{2} w_{2} w_{5}=0, \\
R_{2}\left(w_{1}, w_{2}, w_{3}\right)-y_{4}^{2} w_{1} w_{5}=0,
\end{array}\right.
$$

where $R_{i}\left(w_{1}, w_{2}, w_{3}\right)=R_{i}\left(w_{1}, w_{2}, w_{3} ; \mathbf{y}\right)$ is given by

$$
Q_{i}\left(y_{4} w_{1}, y_{4} w_{2}, y_{4} w_{3},-y_{1} w_{1}-y_{2} w_{2}-y_{3} w_{3}\right)
$$

for $i=1,2$. In particular, this substitution also gives us the model

$$
w_{1} R_{1}\left(w_{1}, w_{2}, w_{3}\right)=w_{2} R_{2}\left(w_{1}, w_{2}, w_{3}\right)
$$

for the non-singular curve $C_{\mathbf{y}}$, so that $S_{\mathbf{y}}$ is the image of the regular map $\phi_{\mathbf{y}}: C_{\mathbf{y}} \rightarrow \mathbb{P}^{3}$ given by

$$
\phi_{\mathbf{y}}\left[w_{1}, w_{2}, w_{3}\right]= \begin{cases}{\left[w_{1}^{2}, w_{1} w_{2}, w_{1} w_{3}, R_{2}\right],} & \left(w_{1}, R_{2}\right) \neq(0,0), \\ {\left[w_{1} w_{2}, w_{2}^{2}, w_{2} w_{3}, R_{1}\right],} & \left(w_{2}, R_{1}\right) \neq(0,0) .\end{cases}
$$

We may therefore conclude that $S_{\mathbf{y}}$ is irreducible for each $\mathbf{y} \in \mathfrak{Y}_{3}$, since $C_{\mathbf{y}}$ is irreducible and $\phi_{\mathbf{y}}$ is a morphism. If we define

and

$$
v_{1}=w_{1}, \quad v_{2}=w_{2}, \quad v_{3}=w_{3}, \quad v_{4}=w_{5}
$$

$$
\Phi_{1}(\mathbf{v})=R_{1}\left(v_{1}, v_{2}, v_{3}\right)-y_{4}^{2} v_{2} v_{4}, \quad \Phi_{2}(\mathbf{v})=R_{2}\left(v_{1}, v_{2}, v_{3}\right)-y_{4}^{2} v_{1} v_{4},
$$

then it remains to count the number of primitive $\mathbf{v} \in \mathbb{Z}^{4}$ such that $\Phi_{1}(\mathbf{v})=$ $\Phi_{2}(\mathbf{v})=0$ and

$$
\left|v_{1}\right| \leq C_{1}, \quad\left|v_{2}\right| \leq C_{2}, \quad\left|v_{3}\right| \leq B, \quad v_{4} \ll B^{2} / \sqrt{C_{1} C_{2}},
$$

by (15) and (17).

Our task is now twofold: we first show that there exists a projection from $S_{\mathbf{y}}$ to $\mathbb{P}^{2}$ which is regular, and then show that it also preserves the expected degree of $S_{\mathbf{y}}$. Since $\mathbf{y} \in \mathfrak{Y}_{3}$, it follows that $\Phi_{1}(0,0,1,0) \neq 0$ by $(21)$ and (25). Thus we deduce that the projection from the point $q=[0,0,1,0] \in \mathbb{P}^{3}$, given by

$$
\pi_{q}: S_{\mathbf{y}} \rightarrow \mathbb{P}^{2}, \quad \pi_{q}\left[v_{1}, v_{2}, v_{3}, v_{4}\right]=\left[v_{1}, v_{2}, v_{4}\right],
$$

is a regular map. 
Turning to the degree of the irreducible curve $\pi_{q}\left(S_{\mathbf{y}}\right)=D_{\mathbf{y}}$ say, we first write $\Phi_{1}$ and $\Phi_{2}$ in the form

$$
\Phi_{i}(\mathbf{v})=a_{i} v_{3}^{2}+b_{i} v_{3}+c_{i}, \quad i=1,2,
$$

for appropriate forms $a_{i}, b_{i}, c_{i} \in \mathbb{Z}\left[v_{1}, v_{2}, v_{4}\right]$. Here $a_{1} \neq 0$ and

$$
c_{1}=r_{1}\left(v_{1}, v_{2}\right)-y_{4}^{2} v_{2} v_{4}, \quad c_{2}=r_{2}\left(v_{1}, v_{2}\right)-y_{4}^{2} v_{1} v_{4}
$$

for certain integral quadratic forms $r_{1}$ and $r_{2}$. In particular, $c_{1}$ and $c_{2}$ are plainly non-zero and are the only coefficients containing terms involving $v_{4}$. But then the image $D_{\mathbf{y}}$ of the projection $\pi_{q}$ is just the zero locus of the resultant of $\Phi_{1}$ and $\Phi_{2}$, given by the determinant

$$
\operatorname{det}\left(\begin{array}{cccc}
a_{1} & b_{1} & c_{1} & 0 \\
0 & a_{1} & b_{1} & c_{1} \\
a_{2} & b_{2} & c_{2} & 0 \\
0 & a_{2} & b_{2} & c_{2}
\end{array}\right) .
$$

We therefore obtain a homogeneous form in $v_{1}, v_{2}, v_{4}$ which contains the non-zero term $a_{1}^{2} c_{2}^{2}$, so that in fact $D_{\mathbf{y}}$ has degree four and contains the monomial $v_{1}^{2} v_{4}^{2}$ with non-zero coefficient, by (28).

In order to estimate the number of rational points on $D_{\mathbf{y}}$, we employ similar techniques to the previous section, although we may now take advantage of our expression for $D_{\mathbf{y}}$ in order to get a sharper lower bound for the quantity $T$, as defined in (3). Thus it is plain that $T \geq C_{1} B^{4} / C_{2}$, since our solutions must lie in the range (26), and the equation for $D_{\mathbf{y}}$ contains the monomial $v_{1}^{2} v_{4}^{2}$ with non-zero coefficient. But then upon applying Lemma 4 , it suffices to sum the contribution

$$
\ll_{\varepsilon} C_{1}^{1 / 16} C_{2}^{3 / 16} B^{1 / 4+\varepsilon}
$$

over all of the vectors $\mathbf{y}$ lying in the range (19). We thereby obtain a total contribution of

$$
\ll_{\varepsilon} C_{1}^{19 / 48} C_{2}^{25 / 48} B^{11 / 12+\varepsilon}
$$

to $\mathcal{L}_{1}(B ; \lambda)$ from those $\mathbf{y} \in \mathfrak{Y}_{3}$.

3.1.3. Completion of the estimate for $\mathcal{L}_{1}(B ; \lambda)$. Combining the estimates (24) and (29), we set $C_{i}=B /\left(2^{e_{i}} \lambda\right)$ for $i=1$, 2 , and sum over every $e_{1}, e_{2} \in \mathbb{Z}$ lying in the range $0 \leq e_{i} \ll \log B$ for which $e_{1}+e_{2} \leq n_{\lambda}$, where $n_{\lambda}$ is chosen so that $2^{n_{\lambda}}<B^{4 / 25} / \lambda^{2} \leq 2^{n_{\lambda}+1}$ for each $\lambda$. In particular therefore, $C_{1}$ and $C_{2}$ lie in the range (16), and with the substitution $C_{i}=B /\left(2^{e_{i}} \lambda\right)$, we have

$$
\sum_{1 \leq \lambda<B^{2 / 25}} \mathcal{L}_{1}(B ; \lambda) \ll_{\varepsilon} \sum_{1 \leq \lambda<B^{2 / 25}}\left(B^{127 / 75+\varepsilon}+\lambda^{-11 / 12} B^{11 / 6+\varepsilon}\right) \ll_{\varepsilon} B^{46 / 25+\varepsilon} .
$$

This is satisfactory for the first half of the proof of Theorem 1 . 
3.2. Estimating $\sum_{\lambda} \mathcal{L}_{2}(B ; \lambda)$ : quadric curves. Recalling from $(7)$ that our original cubic surface $V$ takes the shape

$$
x_{1} Q_{1}(\mathbf{x})=x_{2} Q_{2}(\mathbf{x}),
$$

where the non-singularity of $V$ prevents the possibility that (13) comes to pass, we observe that the projection

$$
\varrho\left[x_{1}, x_{2}, x_{3}, x_{4}\right]= \begin{cases}{\left[x_{1}, x_{2}\right]} & \text { if }\left(x_{1}, x_{2}\right) \neq(0,0), \\ {\left[Q_{2}(\mathbf{x}), Q_{1}(\mathbf{x})\right]} & \text { otherwise }\end{cases}
$$

to the line $x_{1}=x_{2}=0$ defines a morphism $\varrho: V \rightarrow \mathbb{P}^{1}$. We shall be concerned with the fibres of $\varrho$ in our open set $U$, and for any $[a, b] \in \mathbb{P}^{1}$ we see that $\left[a x, b x, x_{3}, x_{4}\right] \in \varrho^{-1}[a, b] \cap U$ only if $x \neq 0$ and

$$
q_{a, b}: \quad a Q_{1}\left(a x, b x, x_{3}, x_{4}\right)=b Q_{2}\left(a x, b x, x_{3}, x_{4}\right) .
$$

It is easy to see that for primitive $\mathbf{x} \in \mathbb{Z}^{4}$ such that $\left(x_{1}, x_{2}\right) \neq(0,0)$, we have $\varrho\left[x_{1}, x_{2}, x_{3}, x_{4}\right]=\left[w_{1}, w_{2}\right]$ in the notation of $(8)$.

In order to estimate $\sum_{\lambda} \mathcal{L}_{2}(B ; \lambda)$, it is therefore enough to bound the number of primitive $\left(x, x_{3}, x_{4}\right) \in \mathbb{Z}^{3}$ lying in the range

$$
|x| \leq \frac{B}{\max \left\{\left|w_{1}\right|,\left|w_{2}\right|\right\}}, \quad\left|x_{3}\right|,\left|x_{4}\right| \leq B,
$$

which lie on the quadric $q_{w_{1}, w_{2}}$, for each primitive integer $\left(w_{1}, w_{2}\right) \in \mathbb{Z}$ such that $\left|w_{1} w_{2}\right| \leq B^{46 / 25}$, by (12). Moreover, it is only the non-singular fibres which contribute anything, since points lying on any reducible $q_{w_{1}, w_{2}}$ of rank at least 1 will lie on a line in the surface $V$, and if $q_{w_{1}, w_{2}}$ vanished identically then $V$ would contain the plane $b x_{1}=a x_{2}$, which is impossible since $V$ is irreducible. The remaining argument is in the vein of Heath-Brown's previously mentioned treatment of cubic surfaces containing three coplanar rational lines [6], although it is considerably simplified in this setting.

Turning to the contribution from the non-singular fibres $q_{w_{1}, w_{2}}$, let $\Delta$ denote the discriminant of the corresponding quadratic form. Thus $\Delta=$ $\left|d\left(w_{1}, w_{2}\right)\right|$, for an appropriate binary integral form $d$ of degree 5 . We deduce from (30) and Lemma 5 that the contribution from each non-singular curve $q_{w_{1}, w_{2}}$ is

$$
\ll_{\varepsilon}\left\{1+\frac{B D^{1 / 2}}{\max \left\{\left|w_{1}\right|,\left|w_{2}\right|\right\}^{1 / 3} \Delta^{1 / 3}}\right\} B^{\varepsilon},
$$

where $D$ is the highest common factor of the $2 \times 2$ minors of the matrix defining $q_{w_{1}, w_{2}}$. We now appeal directly to a result of Heath-Brown [6, Lemma 5], which allows us to make the estimate $D \ll_{V} 1$.

In order to sum (31) over all integers $w_{1}, w_{2}$ such that $\left|w_{1} w_{2}\right| \leq B^{46 / 25}$, we shall consider two different cases according to the size of $\Delta$. We begin however by dividing $\Delta$ into $O(\log B)$ intervals $Z \leq \Delta<2 Z$, and we then 
attain an estimate for each such interval. When $Z>B^{52 / 25}$, we have a contribution

$$
\ll_{\varepsilon, V} \sum_{w_{1}, w_{2}}\left\{1+\frac{B^{23 / 75}}{\max \left\{\left|w_{1}\right|,\left|w_{2}\right|\right\}^{1 / 3}}\right\} B^{\varepsilon} \ll_{\varepsilon, V} B^{46 / 25+\varepsilon} ;
$$

whereas when $Z \leq B^{52 / 25}$, we have the contribution

$$
\ll_{\varepsilon, V} B^{46 / 25+\varepsilon}+\sum_{\left|d\left(w_{1}, w_{2}\right)\right|<2 Z} \frac{B^{1+\varepsilon}}{\max \left\{\left|w_{1}\right|,\left|w_{2}\right|\right\}^{1 / 3} Z^{1 / 3}} .
$$

Factorising the form $d\left(w_{1}, w_{2}\right)$, we note that there exist $\alpha, \beta \in \overline{\mathbb{Q}}$, not both equal to zero, for which $\left|\alpha w_{1}+\beta w_{2}\right| \ll Z^{1 / 5}$ since $d$ has degree 5 and is non-vanishing. Noting that there are $O\left(Z^{1 / 5}\right)$ values of $w_{2}$ for each $w_{1}$ if $\beta \neq 0$ say, we see that

$$
\sum_{\left|d\left(w_{1}, w_{2}\right)\right|<2 Z} \frac{B^{1+\varepsilon}}{\left|w_{1}\right|^{1 / 3} Z^{1 / 3}} \ll \sum_{w_{1}} \frac{B^{1+\varepsilon}}{\left|w_{1}\right|^{1 / 3} Z^{2 / 15}} \ll B^{5 / 3+\varepsilon}
$$

since $Z \geq 1$, so that (33) is bounded by (32). Summing over appropriate powers of 2 , we therefore achieve

$$
\sum_{1 \leq \lambda \leq B} \mathcal{L}_{2}(B ; \lambda) \ll_{\varepsilon, V} B^{46 / 25+\varepsilon},
$$

which is satisfactory for the second half of the proof of Theorem 1 .

4. Non-singular quartic surfaces. Let $B \geq 1$, and let $F \in \mathbb{Z}[\mathbf{x}]$ be the non-singular quartic form defining $W$. Then $\mathcal{N}_{U}(B)$ is the total number of primitive $\mathbf{x} \in \mathbb{Z}^{4}$ lying in the box $\left|x_{i}\right| \leq B(1 \leq i \leq 4)$, such that $F(\mathbf{x})=0$, but $\mathbf{x}$ does not lie on any line contained in the surface. We shall closely follow the above argument for the proof of Theorem 1, and in particular shall use similar notation throughout.

Suppose that the two coplanar rational lines are given by the equations $x_{1}=x_{3}=0$ and $x_{2}=x_{3}=0$. Then $F$ takes the shape

$$
F(\mathbf{x})=x_{1} x_{2} Q(\mathbf{x})-x_{3} C(\mathbf{x}),
$$

where $Q$ and $C$ are integral quadratic and cubic forms respectively. We shall use the special shape that $F$ takes, in order to take out the highest common factor of $x_{1} x_{2}$ and $x_{3}$ in any integer zero $\mathbf{x}$ of $F$. Thus, for each such vector $\mathbf{x}$ we define $\lambda_{1}=\left(x_{1}, x_{3}\right), \lambda_{2}=\left(x_{2}, x_{3} / \lambda_{1}\right)$, and

$$
\mathbf{w}=\left(x_{1} / \lambda_{1}, x_{2} / \lambda_{2}, x_{3} / \lambda_{1} \lambda_{2}, x_{4}\right) .
$$

In particular $\left(w_{1}, w_{3}\right)=\left(w_{2}, w_{3}\right)=1$, and $\mathbf{w}$ lies in the range

$$
\left|w_{1}\right| \leq B / \lambda_{1}, \quad\left|w_{2}\right| \leq B / \lambda_{2}, \quad\left|w_{3}\right| \leq B / \lambda_{1} \lambda_{2}, \quad\left|w_{4}\right| \leq B .
$$


For each $1 \leq \lambda_{1}, \lambda_{2} \leq B$, we turn our attention to the number $\mathcal{M}_{U}\left(B ; \lambda_{1}, \lambda_{2}\right)$ of primitive $\mathbf{w} \in \mathbb{Z}^{4}$ lying in this region for which $F_{\lambda_{1}, \lambda_{2}}(\mathbf{w})=0$, where

$$
\begin{aligned}
F_{\lambda_{1}, \lambda_{2}}= & w_{1} w_{2} Q\left(\lambda_{1} w_{1}, \lambda_{2} w_{2}, \lambda_{1} \lambda_{2} w_{3}, w_{4}\right) \\
& -w_{3} C\left(\lambda_{1} w_{1}, \lambda_{2} w_{2}, \lambda_{1} \lambda_{2} w_{3}, w_{4}\right),
\end{aligned}
$$

and $\mathbf{w}$ does not lie on any line contained in the surface.

In order to estimate the quantity $\mathcal{M}_{U}\left(B ; \lambda_{1}, \lambda_{2}\right)$, we split our considerations according to the size of the variables $w_{1}, w_{2}, w_{3}$. Thus for given $\theta \in \mathbb{Q}^{*}$, let $\mathcal{L}_{1}^{\theta}\left(B ; \lambda_{1}, \lambda_{2}\right)$ denote the number of vectors $\mathbf{w}$ counted by $\mathcal{M}_{U}\left(B ; \lambda_{1}, \lambda_{2}\right)$ for which

$$
\left|\lambda_{2} w_{1} w_{3}\right|>B^{\theta} \text { and }\left|\lambda_{1} w_{2} w_{3}\right|>B^{\theta},
$$

and let $\mathcal{L}_{2}^{\theta}\left(B ; \lambda_{1}, \lambda_{2}\right)$ denote the corresponding number of $\mathbf{w}$ for which

$$
\left|\lambda_{2} w_{1} w_{3}\right| \leq B^{\theta} \quad \text { or } \quad\left|\lambda_{1} w_{2} w_{3}\right| \leq B^{\theta} .
$$

In order to also obtain an unconditional version of Theorem 2 , we defer our choice of $\theta \leq 2$ until the end of Section 4.2, although we henceforth assume that $\theta \geq 3 / 2$. For any choice of $\theta \in \mathbb{Q}^{*}$ however, we clearly have the estimate

$$
\mathcal{N}_{U}(B) \leq \sum_{\lambda_{1} \lambda_{2}<B^{2-\theta}} \mathcal{L}_{1}^{\theta}\left(B ; \lambda_{1}, \lambda_{2}\right)+\sum_{\lambda_{1}, \lambda_{2} \leq B} \mathcal{L}_{2}^{\theta}\left(B ; \lambda_{1}, \lambda_{2}\right),
$$

since only those $\lambda_{1}, \lambda_{2}$ for which $\lambda_{1} \lambda_{2}<B^{2-\theta}$ will yield a non-zero value of $\mathcal{L}_{1}^{\theta}\left(B ; \lambda_{1}, \lambda_{2}\right)$.

4.1. Estimating $\sum_{\lambda_{1}, \lambda_{2}} \mathcal{L}_{1}^{\theta}\left(B ; \lambda_{1}, \lambda_{2}\right)$. For ease of notation we first absorb each appearance of $\lambda_{1}$ and $\lambda_{2}$ into the coefficients of $Q$ and $C$ in $W_{\lambda_{1}, \lambda_{2}}$, where $W_{\lambda_{1}, \lambda_{2}}$ denotes the surface $F_{\lambda_{1}, \lambda_{2}}=0$. There can be no point $\left[\eta_{1}, 0,0, \eta_{4}\right] \in \mathbb{P}^{3}$ for which

$$
\eta_{1} Q\left(\eta_{1}, 0,0, \eta_{4}\right)=C\left(\eta_{1}, 0,0, \eta_{4}\right)=0,
$$

since it would produce a singularity in the surface $W_{\lambda_{1}, \lambda_{2}}$. Similarly, there can be no point $\left[0, \eta_{2}, 0, \eta_{4}\right] \in \mathbb{P}^{3}$ for which

$$
\eta_{2} Q\left(0, \eta_{2}, 0, \eta_{4}\right)=C\left(0, \eta_{2}, 0, \eta_{4}\right)=0 .
$$

In particular we note that

$$
C(0,0,0,1) \neq 0 \text {. }
$$

Our primary task in this section will be to estimate the number of primitive $\mathbf{w} \in \mathbb{Z}^{4}$ such that $F_{\lambda_{1}, \lambda_{2}}(\mathbf{w})=0, \mathbf{w}$ does not lie on any line in the surface, and for which

$$
C_{1} / 2<\left|w_{1}\right| \leq C_{1}, \quad C_{2} / 2<\left|w_{2}\right| \leq C_{2}, \quad C_{3} / 2<\left|w_{3}\right| \leq C_{3}, \quad\left|w_{4}\right| \leq B,
$$
where

$$
C_{1} C_{3} \gg \frac{B^{\theta}}{\lambda_{2}}, \quad C_{2} C_{3} \gg \frac{B^{\theta}}{\lambda_{1}}
$$


and

$$
1 \leq C_{1} \leq \frac{B}{\lambda_{1}}, \quad 1 \leq C_{2} \leq \frac{B}{\lambda_{2}}, \quad 1 \leq C_{3} \leq \frac{B}{\lambda_{1} \lambda_{2}} .
$$

We shall ultimately sum our bounds for this quantity over powers of 2 , in order to get an estimate for $\mathbf{w}$ lying in the general range defined by (35) and (36) for each $\lambda_{1}, \lambda_{2}$, and so an estimate for $\mathcal{L}_{1}^{\theta}\left(B ; \lambda_{1}, \lambda_{2}\right)$.

As in the case of cubic surfaces containing one rational line, we begin with an observation which allows us to switch the problem to an irreducible surface of higher degree. Since $w_{1} w_{2}$ and $w_{3}$ are coprime for each vector counted by $\mathcal{L}_{1}^{\theta}\left(B ; \lambda_{1}, \lambda_{2}\right)$, it follows that there exists $w_{5} \in \mathbb{Z}$ such that

$$
Q\left(w_{1}, w_{2}, w_{3}, w_{4}\right)=w_{3} w_{5}, \quad C\left(w_{1}, w_{2}, w_{3}, w_{4}\right)=w_{1} w_{2} w_{5},
$$

where $C(0,0,0,1) \neq 0$ by (40). We denote this intersection of hypersurfaces in $\mathbb{P}^{4}$ by $T=T_{\lambda_{1}, \lambda_{2}}$, and observe that

$$
w_{5} \ll \min \left\{\frac{B^{2}}{C_{3}}, \frac{B^{3}}{C_{1} C_{2}}\right\},
$$

since $C_{1}, C_{2}, C_{3} \ll B$. The variety $T$ is the image of $W_{\lambda_{1}, \lambda_{2}}$ under the map

$$
\begin{aligned}
\psi\left[w_{1}, w_{2}, w_{3}, w_{4}\right] & \\
= & \begin{cases}{\left[w_{1} w_{3}, w_{2} w_{3}, w_{3}^{2}, w_{3} w_{4}, Q\right],} & \left(w_{3}, Q\right) \neq(0,0), \\
{\left[w_{1}^{2} w_{2}, w_{1} w_{2}^{2}, w_{1} w_{2} w_{3}, w_{1} w_{2} w_{4}, C\right],} & \left(w_{1} w_{2}, C\right) \neq(0,0) .\end{cases}
\end{aligned}
$$

This is analogous to (18). We use the non-singularity of $W_{\lambda_{1}, \lambda_{2}}$ to deduce, via (38) and (39), that $\psi: W_{\lambda_{1}, \lambda_{2}} \rightarrow \mathbb{P}^{4}$ is a regular map with image $T$. Moreover, we have a corresponding isomorphism of open sets $W_{\lambda_{1}, \lambda_{2}}-L \cong$ $T-\left\{p_{0}\right\}$, where $L$ is the union of lines $w_{i}=w_{3}=0$ for $i=1,2$, and $p_{0}=[0,0,0,0,1]$.

Proceeding as in the case of cubic surfaces, we use Lemma 1 to take plane sections of the quartic surface $W_{\lambda_{1}, \lambda_{2}}$, recalling that each $\mathbf{w}$ lies in the range (41). Therefore for each primitive non-zero $\mathbf{y} \in \mathbb{Z}^{4}$ lying in the range

$$
y_{i} \ll \begin{cases}C_{i}^{-1}\left(C_{1} C_{2} C_{3} B\right)^{1 / 3}, & i=1,2,3, \\ B^{-2 / 3}\left(C_{1} C_{2} C_{3}\right)^{1 / 3}, & i=4,\end{cases}
$$

it suffices to estimate the number of primitive integer $\mathbf{w}$ in the range (41) which lie on the curve

$$
C_{\mathbf{y}}: \quad \mathbf{w} \cdot \mathbf{y}=F_{\lambda_{1}, \lambda_{2}}(\mathbf{w})=0,
$$

but not on any linear component of it. As above, our key saving over previous approaches will come from the treatment of the non-singular $C_{\mathbf{y}}$, for which we translate our attention to the corresponding hyperplane section of $T \subset \mathbb{P}^{4}$. 
Before continuing we take a moment to establish that each of the bounds on the right hand side of (45) is $\gg 1$. But this is simply a matter of applying the lower bounds (42) for $C_{1} C_{3}$ and $C_{2} C_{3}$, together with the upper bound $C_{3} \leq B /\left(\lambda_{1} \lambda_{2}\right)$. For example, we see that

$$
C_{1} C_{2} C_{3} \gg \frac{B^{2 \theta}}{\lambda_{1} \lambda_{2} C_{3}} \geq B^{2 \theta-1} \geq B^{2}
$$

since $\theta \geq 3 / 2$, from which it follows that $B^{-2 / 3}\left(C_{1} C_{2} C_{3}\right)^{1 / 3} \gg 1$, as required.

We begin by decomposing all of the primitive $\mathbf{y} \in \mathbb{Z}^{4}$ into three disjoint sets $\mathfrak{Y}_{1} \cup \mathfrak{Y}_{2} \cup \mathfrak{Y}_{3}$. Thus we let $\mathfrak{Y}_{1}$ denote the subset of $\mathbf{y}$ for which $C_{\mathbf{y}}$ is singular, and let $\mathfrak{Y}_{2}$ be the subset of $\mathbf{y} \notin \mathfrak{Y}_{1}$ for which

$$
y_{4} C\left(0,0, y_{4},-y_{3}\right)=0 \text {. }
$$

Finally, we let $\mathfrak{Y}_{3}$ be the remaining set of integer $\mathbf{y}$. It follows from (40) that the form $C\left(0,0, y_{4},-y_{3}\right)$ is not identically zero as a function of $y_{3}, y_{4}$, and so Lemma 2 implies that the set $\mathfrak{Y}_{2}$ contains at most $O(B)$ vectors $\mathbf{y}$ lying in the range (45).

4.1.1. Contribution from the case $\mathbf{y} \in \mathfrak{Y}_{1} \cup \mathfrak{Y}_{2}$. In order to estimate the contribution from the $\mathbf{y} \in \mathfrak{Y}_{1}$, we suppose that $C_{i} \leq C_{j} \leq C_{k}$ for some triple $\{i, j, k\}=\{1,2,3\}$. We apply the theory of the dual surface, as in Section 3.1.1, to deduce via Lemma 3 that there are at most

$$
\ll_{\varepsilon} \frac{\left(C_{1} C_{2} C_{3} B\right)^{2 / 3}}{C_{i} C_{j}} B^{\varepsilon}
$$

vectors $\mathbf{y}$ lying in the range (45) for which $C_{\mathbf{y}}$ is singular. If $y_{i} \neq 0$, we may apply the bounds of Lemma 4 to the quartic form

$$
g_{\mathbf{y}}\left(w_{j}, w_{k}, w_{4}\right)=F_{\lambda_{1}, \lambda_{2}}\left(-y_{j} w_{j}-y_{k} w_{k}-y_{4} w_{4}, y_{i} w_{j}, y_{i} w_{k}, y_{i} w_{4}\right) .
$$

Since we are to ignore points lying on any linear factors of $g_{\mathbf{y}}$, it suffices to consider the contribution from the irreducible factors of degree three and four from all of the vectors $\mathbf{y} \in \mathfrak{Y}_{1}$. Indeed, we observe via a result of Colliot-Thélène [8, Appendix] that a non-singular quartic surface contains finitely many curves of degree $\leq 2$, so that the quadratic factors contribute just $O_{\varepsilon}\left(B^{1+\varepsilon}\right)$ to $\mathcal{L}_{1}^{\theta}\left(B ; \lambda_{1}, \lambda_{2}\right)$, by Lemma 4 and the fact that $\mathbf{w}$ is contained in a cube of side $2 B$.

Turning to the factors of higher degree, it suffices to sum over all $\mathbf{y} \in \mathfrak{Y}_{1}$ the contribution

$$
\ll_{\varepsilon}\left(B C_{j}\right)^{1 / 3+\varepsilon}
$$

from such factors, by (5) and the ranges (41) for $w_{j}, w_{k}, w_{4}$. Applying the bound (47) for the number of such $\mathbf{y}$, we therefore obtain the contribution

$$
\ll_{\varepsilon} B^{1+\varepsilon} C_{k}^{2 / 3} C_{i}^{-1 / 3}
$$


to $\mathcal{L}_{1}^{\theta}\left(B ; \lambda_{1}, \lambda_{2}\right)$ from those $\mathbf{y} \in \mathfrak{Y}_{1}$ for which $y_{i} \neq 0$. We clearly get the same contribution had we instead assumed that $y_{j}$ or $y_{k}$ were non-zero. When $i=1$ this is equal to

$$
\begin{aligned}
B^{1+\varepsilon} C_{k}^{2 / 3} C_{3}^{1 / 3}\left(C_{1} C_{3}\right)^{-1 / 3} & \ll_{\varepsilon} \lambda_{2}^{1 / 3} B^{1-\theta / 3+\varepsilon} C_{k}^{2 / 3} C_{3}^{1 / 3} \\
& \ll_{\varepsilon}\left(\lambda_{1} \lambda_{2}\right)^{-1 / 3} B^{2-\theta / 3+\varepsilon},
\end{aligned}
$$

by (42) and (43). One argues identically for the case $i=2$, and similarly when $i=3$ one has

$$
\begin{aligned}
B^{1+\varepsilon} C_{k}^{2 / 3} C_{i}^{-1 / 3} & =B^{1+\varepsilon} C_{j}^{1 / 3} C_{k}^{2 / 3}\left(C_{j} C_{3}\right)^{-1 / 3} \\
& \ll_{\varepsilon} \lambda_{k}^{1 / 3} B^{1-\theta / 3+\varepsilon} C_{j}^{1 / 3} C_{k}^{2 / 3}
\end{aligned}
$$

which is satisfactorily bounded by (48).

Turning to the contribution from the $\mathbf{y} \in \mathfrak{Y}_{2}$, we recall that each $C_{\mathbf{y}}$ is non-singular and $\mathbf{w}$ is contained in the region (41). Suppose first that $y_{4} \neq 0$, so that upon eliminating $w_{4}$ we may apply the bound (4) of Lemma 4 to a certain non-singular quartic form $g_{\mathbf{y}}\left(w_{1}, w_{2}, w_{3}\right)$, say. Summing over the $O(B)$ possible $\mathbf{y} \in \mathfrak{Y}_{2}$ lying in the range (45), we obtain a contribution of $O_{\varepsilon}\left(\left(C_{1} C_{2} C_{3}\right)^{1 / 6} B^{1+\varepsilon}\right)$ from those $\mathbf{y}$ for which $y_{4} \neq 0$. Arguing similarly, we obtain the contributions $O_{\varepsilon}\left(\left(C_{2} C_{3}\right)^{1 / 6} B^{7 / 6+\varepsilon}\right)$ from the case $y_{1} \neq 0$ and $O_{\varepsilon}\left(\left(C_{1} C_{3}\right)^{1 / 6} B^{7 / 6+\varepsilon}\right)$ from the case $y_{2} \neq 0$, so that we therefore obtain a total contribution of

$$
\ll_{\varepsilon} \max \left\{C_{1}, C_{2}\right\}^{1 / 6} C_{3}^{1 / 6} B^{7 / 6+\varepsilon}
$$

to $\mathcal{L}_{1}^{\theta}\left(B ; \lambda_{1}, \lambda_{2}\right)$ from the set $\mathfrak{Y}_{2} ;$ indeed, the contribution from the $O(1)$ vectors $\mathbf{y} \in \mathfrak{Y}_{2}$ for which $y_{1}=y_{2}=y_{4}=0$ is clearly satisfactory.

4.1.2. Contribution from the case $\mathbf{y} \in \mathfrak{Y}_{3}$. Arguing as in Section 3.1.2, we see that for each $\mathbf{y} \in \mathfrak{Y}_{3}$ lying in the range (45) it suffices to estimate the number of primitive $\mathbf{w} \in \mathbb{Z}^{5}$ lying in the region defined by (41) and (44) which lie on the curve $S_{\mathbf{y}}=H_{\mathbf{y}} \cap T \subset \mathbb{P}^{4}$. Since $y_{4} \neq 0$ for all $\mathbf{y} \in \mathfrak{Y}_{3}$, we eliminate $w_{4}$ from the equations defining $S_{\mathbf{y}}$ to obtain the explicit model

$$
S_{\mathbf{y}}:\left\{\begin{array}{l}
q\left(w_{1}, w_{2}, w_{3}\right)-y_{4}^{2} w_{3} w_{5}=0, \\
c\left(w_{1}, w_{2}, w_{3}\right)-y_{4}^{3} w_{1} w_{2} w_{5}=0
\end{array}\right.
$$

where $q$ and $c$ are given by

$$
\begin{aligned}
& q\left(w_{1}, w_{2}, w_{3}\right)=Q\left(y_{4} w_{1}, y_{4} w_{2}, y_{4} w_{3},-y_{1} w_{1}-y_{2} w_{2}-y_{3} w_{3}\right), \\
& c\left(w_{1}, w_{2}, w_{3}\right)=C\left(y_{4} w_{1}, y_{4} w_{2}, y_{4} w_{3},-y_{1} w_{1}-y_{2} w_{2}-y_{3} w_{3}\right) .
\end{aligned}
$$

In particular, this substitution also gives us the model

$$
y_{4} w_{1} w_{2} q\left(w_{1}, w_{2}, w_{3}\right)=w_{3} c\left(w_{1}, w_{2}, w_{3}\right)
$$


for the non-singular curve $C_{\mathbf{y}}$. Therefore $S_{\mathbf{y}}$ is the image of the map $\psi_{\mathbf{y}}$ : $C_{\mathbf{y}} \rightarrow \mathbb{P}^{3}$ given by

$$
\psi_{\mathbf{y}}\left[w_{1}, w_{2}, w_{3}\right]= \begin{cases}{\left[w_{1} w_{3}, w_{2} w_{3}, w_{3}^{2}, q\right],} & \left(w_{3}, q\right) \neq(0,0), \\ {\left[w_{1}^{2} w_{2}, w_{1} w_{2}^{2}, w_{1} w_{2} w_{3}, c\right],} & \left(w_{1} w_{2}, c\right) \neq(0,0) .\end{cases}
$$

Since (38) and (39) ensure that $\psi_{\mathbf{y}}$ is a regular map, it follows that $S_{\mathbf{y}}$ is irreducible for each $\mathbf{y} \in \mathfrak{Y}_{3}$. If we define

$$
v_{1}=w_{1}, \quad v_{2}=w_{2}, \quad v_{3}=w_{3}, \quad v_{4}=w_{5}
$$

and

$$
\Phi(\mathbf{v})=q\left(v_{1}, v_{2}, v_{3}\right)-y_{4}^{2} v_{3} v_{4}, \quad \Psi(\mathbf{v})=c\left(v_{1}, v_{2}, v_{3}\right)-y_{4}^{3} v_{1} v_{2} v_{4},
$$

then it suffices to count the number of primitive $\mathbf{v} \in \mathbb{Z}^{4}$ such that $\Phi(\mathbf{v})=$ $\Psi(\mathbf{v})=0$ and

$$
\left|v_{1}\right| \leq C_{1}, \quad\left|v_{2}\right| \leq C_{2}, \quad\left|v_{3}\right| \leq C_{3}, \quad v_{4} \ll \min \left\{\frac{B^{2}}{C_{3}}, \frac{B^{3}}{C_{1} C_{2}}\right\},
$$

by (41) and (44).

In order to be able to count points on the curve $S_{\mathbf{y}}$, we first project to $\mathbb{P}^{2}$ in such a way that the image is irreducible and of degree six. Since $\mathbf{y} \in \mathfrak{Y}_{3}$, it follows that $\Psi(0,0,1,0)=c(0,0,1) \neq 0$ by $(50)$ and the fact that (46) does not occur for $\mathbf{y} \in \mathfrak{Y}_{3}$. Therefore the projection (27) from the point $q=[0,0,1,0]$ is a regular map, and its image $\pi_{q}\left(S_{\mathbf{y}}\right)=D_{\mathbf{y}}$, say, is an irreducible plane curve. Writing

$$
\Phi(\mathbf{v})=a_{0} v_{3}^{2}+a_{1} v_{3}+a_{2}, \quad \Psi(\mathbf{v})=b_{0} v_{3}^{3}+b_{1} v_{3}^{2}+b_{2} v_{3}+b_{3}
$$

for $a_{i}, b_{i} \in \mathbb{Z}\left[v_{1}, v_{2}, v_{4}\right]$, we note that $b_{0}=\Psi(0,0,1,0) \neq 0$ and

$$
a_{1}=l\left(v_{1}, v_{2}\right)-y_{4}^{2} v_{4}, \quad b_{3}=r\left(v_{1}, v_{2}\right)-y_{4}^{3} v_{1} v_{2} v_{4}
$$

for certain integral linear and cubic forms $l$ and $r$ respectively. In particular, $a_{1}$ and $b_{3}$ are plainly non-zero and are the only coefficients containing terms involving $v_{4}$. Each $a_{i}, b_{i} \in \mathbb{Z}\left[v_{1}, v_{2}, v_{4}\right]$ is a form of degree $i$, and by considering the resultant of $\Phi$ and $\Psi$,

$$
\operatorname{det}\left(\begin{array}{ccccc}
a_{0} & a_{1} & a_{2} & 0 & 0 \\
0 & a_{0} & a_{1} & a_{2} & 0 \\
0 & 0 & a_{0} & a_{1} & a_{2} \\
b_{0} & b_{1} & b_{2} & b_{3} & 0 \\
0 & b_{0} & b_{1} & b_{2} & b_{3}
\end{array}\right)
$$

we obtain a homogeneous form in $v_{1}, v_{2}, v_{4}$ which contains in particular the non-zero term $a_{1}^{3} b_{0} b_{3}$. Thus we deduce that $D_{\mathbf{y}}$ has degree six and contains the monomial $v_{1} v_{2} v_{4}^{4}$ with non-zero coefficient, by (52). We recall the definition (3) of $T$, which together with the ranges (51) for our solutions is 
equal to

$$
T=\max \left\{C_{1}^{f_{1}} C_{2}^{f_{2}} \min \left\{\frac{B^{2}}{C_{3}}, \frac{B^{3}}{C_{1} C_{2}}\right\}^{f_{3}}\right\},
$$

where the maximum is taken over all non-negative integer triples $\left(f_{1}, f_{2}, f_{3}\right)$ such that $f_{1}+f_{2}+f_{3}=6$, for which the corresponding monomial $v_{1}^{f_{1}} v_{2}^{f_{2}} v_{4}^{f_{3}}$ has a non-zero coefficient in $D_{\mathbf{y}}$. Taking $\left(f_{1}, f_{2}, f_{3}\right)=(1,1,4)$, we deduce that

$$
T \geq \begin{cases}C_{1} C_{2} C_{3}^{-4} B^{8}, & C_{1} C_{2}<B C_{3}, \\ \left(C_{1} C_{2}\right)^{-3} B^{12}, & C_{1} C_{2} \geq B C_{3} .\end{cases}
$$

In order to estimate the total number of rational points lying on $D_{\mathbf{y}}$ in the region (51), we apply the lower bound (53) for the quantity $T$ in Lemma 4. Thus it suffices to sum the contribution

$$
\ll_{\varepsilon} \begin{cases}\left(C_{1} C_{2}\right)^{5 / 36} C_{3}^{-1 / 18} B^{1 / 9+\varepsilon}, & C_{1} C_{2}<B C_{3}, \\ \left(C_{1} C_{2}\right)^{1 / 12} B^{1 / 6+\varepsilon}, & C_{1} C_{2} \geq B C_{3},\end{cases}
$$

over all of the $O\left(\left(C_{1} C_{2} C_{3} B\right)^{1 / 3}\right)$ vectors $\mathbf{y}$ lying in the range (45). We thereby obtain a total contribution of

$$
\ll_{\varepsilon} \begin{cases}\left(C_{1} C_{2}\right)^{17 / 36} C_{3}^{5 / 18} B^{4 / 9+\varepsilon}, & C_{1} C_{2}<B C_{3}, \\ \left(C_{1} C_{2}\right)^{5 / 12} C_{3}^{1 / 3} B^{1 / 2+\varepsilon}, & C_{1} C_{2} \geq B C_{3},\end{cases}
$$

to $\mathcal{L}_{1}^{\theta}\left(B ; \lambda_{1}, \lambda_{2}\right)$ from the set $\mathfrak{Y}_{3}$.

4.1.3. Completion of the estimate for $\mathcal{L}_{1}^{\theta}\left(B ; \lambda_{1}, \lambda_{2}\right)$. Choose integers $n_{1}$ and $n_{2}$ such that

$$
2^{n_{1}}<B^{2-\theta} / \lambda_{1}^{2} \leq 2^{n_{1}+1}, \quad 2^{n_{2}}<B^{2-\theta} / \lambda_{2}^{2} \leq 2^{n_{2}+1}
$$

for each $\lambda_{1}$ and $\lambda_{2}$, and set

$$
C_{1}=\frac{B}{2^{e_{1}} \lambda_{1}}, \quad C_{2}=\frac{B}{2^{e_{2}} \lambda_{2}}, \quad C_{3}=\frac{B}{2^{e_{3}} \lambda_{1} \lambda_{2}}
$$

in the estimates (48), (49) and (54). We then sum over every $e_{1}, e_{2}, e_{3} \in \mathbb{Z}$ lying in the range $0 \leq e_{i} \ll \log B$, for which $e_{1}+e_{3} \leq n_{1}$ and $e_{2}+e_{3} \leq n_{2}$, so that in particular $C_{1}, C_{2}, C_{3}$ lie in the ranges defined by (42) and (43). We therefore deduce that $\sum_{\lambda_{1}, \lambda_{2}} \mathcal{L}_{1}^{\theta}\left(B ; \lambda_{1}, \lambda_{2}\right)$ is at most

$$
\begin{aligned}
& \ll_{\varepsilon} B^{\varepsilon} \sum_{\lambda_{1} \lambda_{2}<B^{2-\theta}}\left\{\frac{B^{2-\theta / 3}}{\left(\lambda_{1} \lambda_{2}\right)^{1 / 3}}+\frac{B^{3 / 2}}{\min \left\{\lambda_{1} \lambda_{2}^{2}, \lambda_{1}^{2} \lambda_{2}\right\}^{1 / 6}}+\frac{B^{5 / 3}}{\left(\lambda_{1} \lambda_{2}\right)^{3 / 4}}\right\} \\
& \ll_{\varepsilon} B^{\varepsilon}\left\{B^{10 / 3-\theta}+B^{19 / 6-5 \theta / 6}+B^{13 / 6-\theta / 4}\right\} \\
& \ll_{\varepsilon} \begin{cases}B^{19 / 6-5 \theta / 6+\varepsilon} & \text { if } \theta \leq 12 / 7, \\
B^{13 / 6-\theta / 4+\varepsilon} & \text { if } \theta>12 / 7,\end{cases}
\end{aligned}
$$

since $2 \geq \theta \geq 3 / 2$. 
4.2. Estimating $\sum_{\lambda_{1}, \lambda_{2}} \mathcal{L}_{2}^{\theta}\left(B ; \lambda_{1}, \lambda_{2}\right)$ : cubic curves. With Section 3.2 as our template, we recall the shape $x_{1} x_{2} Q(\mathbf{x})=x_{3} C(\mathbf{x})$ that our original quartic surface $W$ takes, and consider the projections

$$
\begin{aligned}
& \varrho_{1}\left[x_{1}, x_{2}, x_{3}, x_{4}\right]= \begin{cases}{\left[x_{1}, x_{3}\right],} & \left(x_{1}, x_{3}\right) \neq(0,0), \\
{\left[C(\mathbf{x}), x_{2} Q(\mathbf{x})\right],} & \left(C, x_{2} Q\right) \neq(0,0),\end{cases} \\
& \varrho_{2}\left[x_{1}, x_{2}, x_{3}, x_{4}\right]= \begin{cases}{\left[x_{2}, x_{3}\right],} & \left(x_{2}, x_{3}\right) \neq(0,0), \\
{\left[C(\mathbf{x}), x_{1} Q(\mathbf{x})\right],} & \left(C, x_{1} Q\right) \neq(0,0) .\end{cases}
\end{aligned}
$$

Since the non-singularity of $W$ prevents the possibility that (38) or (39) comes to pass, we see that $\varrho_{1}, \varrho_{2}: W \rightarrow \mathbb{P}^{1}$ are both regular maps. We suppose without loss of generality that $\left|\lambda_{2} w_{1} w_{3}\right| \leq B^{\theta}$ in (37), and so consider the map $\varrho_{1}$. We shall be concerned with the fibres of $\varrho_{1}: W \rightarrow \mathbb{P}^{1}$ in our open set $U$. Thus for any $[a, b] \in \mathbb{P}^{1}$ we see that $\left[a x, x_{2}, b x, x_{4}\right] \in \varrho_{1}^{-1}[a, b] \cap U$ only if $x \neq 0$ and

$$
c_{a, b}: \quad a x_{2} Q\left(a x, x_{2}, b x, x_{4}\right)=b C\left(a x, x_{2}, b x, x_{4}\right) .
$$

Arguing as previously, it is easy to see that for primitive integer vectors $\mathbf{x}$ such that $\left(x_{1}, x_{3}\right) \neq(0,0)$, we have $\varrho_{1}\left[x_{1}, x_{2}, x_{3}, x_{4}\right]=\left[w_{1}, \lambda_{2} w_{3}\right]$ in the notation of (34). Thus in order to estimate $\sum_{\lambda_{1}, \lambda_{2}} \mathcal{L}_{2}^{\theta}\left(B ; \lambda_{1}, \lambda_{2}\right)$, it is enough to bound the number of primitive $\left(x, x_{2}, x_{4}\right) \in \mathbb{Z}^{3}$ lying in the range

$$
|x| \leq \frac{B}{\max \{|a|,|b|\}}, \quad\left|x_{2}\right|,\left|x_{4}\right| \leq B,
$$

which lie on the cubic curve $c_{a, b}$, for each primitive integer $(a, b) \in \mathbb{Z}$ such that $|a|,|b| \leq B$ and $|a b|=\left|\lambda_{2} w_{1} w_{3}\right| \leq B^{\theta}$, by (37).

We shall need some control over those $a, b \in \mathbb{Z}$ which give rise to singular fibres $c_{a, b}$. However elimination theory produces a system of polynomials $E_{i} \in \overline{\mathbb{Q}}[a, b]$ for $1 \leq i \ll 1$ which have a non-zero solution over $\overline{\mathbb{Q}}$ if and only if the three partial derivatives of $c_{a, b}$ have one. Moreover, each $E_{i}$ will have total degree at most $O(1)$ (depending only upon the degree of the partial derivatives), although it does not necessarily follow that the $E_{i}$ are non-zero. However, it is a familiar geometric fact that for any non-singular projective variety $X$ defined over a field of characteristic zero, the generic fibre of a morphism $f: X \rightarrow \mathbb{P}^{1}$ is non-singular. Thus we may assume that there exists at least one non-zero polynomial $E_{k}(a, b)$ of degree $d \ll 1$, which vanishes whenever the fibre $c_{a, b}$ is singular. But $E_{k}=0$ defines an affine plane curve, to which we can apply the bound (6) attached to Lemma 4, and so deduce the following result.

Lemma 7. For given $R \geq 1$, there are at most $O_{\varepsilon}\left(R^{1+\varepsilon}\right)$ integer points $a, b$ of size $|a|,|b| \leq R$ for which the fibre $\varrho_{1}^{-1}[a, b]$ is singular. 
Handling the case of singular fibres first, we note immediately that any linear components of $c_{a, b}$ will correspond to lines in the surface $W$, and so will contribute nothing to $\sum_{\lambda_{1}, \lambda_{2}} \mathcal{L}_{2}^{\theta}\left(B ; \lambda_{1}, \lambda_{2}\right)$. Whenever $c_{a, b}$ has an irreducible quadratic component, we note that its equation must contain some non-zero monomial which does not contain $x$ as a factor. Thus we may apply Lemma 4 with $T \geq B^{2}$, in conjunction with the ranges (56) for $\left(x, x_{2}, x_{4}\right)$, to get a contribution of

$$
\ll_{\varepsilon} \sum_{a, b} \frac{B^{1+\varepsilon}}{\max \{|a|,|b|\}^{1 / 2}} .
$$

Dividing each of $a$ and $b$ into $O(\log B)$ intervals, we use Lemma 7 to get an estimate for the contribution from each interval, and then sum the resulting bound over powers of 2 to obtain a total contribution of $O_{\varepsilon}\left(B^{3 / 2+\varepsilon}\right)$ to $\sum_{\lambda_{1}, \lambda_{2}} \mathcal{L}_{2}^{\theta}\left(B ; \lambda_{1}, \lambda_{2}\right)$, which is satisfactory. One proceeds similarly in order to estimate the contribution from the possibility that $c_{a, b}$ is a singular irreducible cubic curve.

Turning to the case of non-singular $c_{a, b}$, we apply two alternative upper bounds in order to deduce Theorem 2 and a corresponding unconditional version, respectively. Beginning with the second of these - for which in fact Lemma 7 can be made redundant - we note (as in the handling of the singular fibres above) that we may take the lower bound $T \geq B^{3}$ in Lemma 4 . This thereby gives a contribution of

$$
\ll_{\varepsilon} \sum_{|a b| \leq B^{\theta}} \frac{B^{2 / 3+\varepsilon}}{\max \{|a|,|b|\}^{1 / 3}} \ll_{\varepsilon} \sum_{|a b| \leq B^{\theta}} \frac{B^{2 / 3+\varepsilon}}{|a b|^{1 / 6}} \ll_{\varepsilon} B^{2 / 3+5 \theta / 6+\varepsilon}
$$

to $\sum_{\lambda_{1}, \lambda_{2}} \mathcal{L}_{2}^{\theta}\left(B ; \lambda_{1}, \lambda_{2}\right)$, by (56). Combining this with (55), we take the value $\theta=3 / 2$ in the two bounds, and so deduce the unconditional result

$$
\mathcal{N}_{U}(B)=O_{\varepsilon, W}\left(B^{23 / 12+\varepsilon}\right) .
$$

This is distinctly weaker than Heath-Brown's previously mentioned bound for non-singular quartic surfaces, where the exponent was $16 / 9+\varepsilon$.

Turning finally to the completion of the proof of Theorem 2, we may clearly assume that $c_{a, b}$ is an elliptic curve, since otherwise $c_{a, b}(\mathbb{Q})$ is empty. Applying Lemma 6, and upon assuming the so-called rank hypothesis (2), we therefore deduce the overall contribution

$$
\ll_{\varepsilon} \sum_{|a b| \leq B^{\theta}} B^{\varepsilon}\left\|c_{a, b}\right\|^{\varepsilon} \ll_{\varepsilon, W} B^{\theta+\varepsilon}
$$

to $\sum_{\lambda_{1}, \lambda_{2}} \mathcal{L}_{2}^{\theta}\left(B ; \lambda_{1}, \lambda_{2}\right)$. Taking the value $\theta=26 / 15$, this completes the proof of Theorem 2 once taken in conjunction with the bound (55). 


\section{References}

[1] E. Bombieri and J. Pila, The number of integral points on arcs and ovals, Duke Math. J. 59 (1989), 337-357.

[2] T. D. Browning, Sums of four biquadrates, Math. Proc. Cambridge Philos. Soc. 134 (2003), 385-395.

[3] -, Counting rational points on curves and surfaces, $\mathrm{PhD}$ thesis, Oxford Univ., 2001.

[4] J. Franke, Yu. I. Manin and Yu. Tschinkel, Rational points of bounded height on Fano varieties, Invent. Math. 95 (1989), 421-435.

[5] J. Harris, Algebraic Geometry, Springer, 1992.

[6] D. R. Heath-Brown, The density of rational points on cubic surfaces, Acta Arith. 79 (1997), 17-30.

[7] -, Counting rational points on cubic surfaces, Astérisque 251 (1998), 13-29.

[8] - , The density of rational points on curves and surfaces, Ann. of Math. 155 (2002), $553-595$.

[9] C. Hooley, On the numbers that are representable as the sum of two cubes, J. Reine Angew. Math. 314 (1980), 146-173.

[10] -, On another sieve method and the numbers that are a sum of two hth powers: II, ibid. 475 (1996), 55-75.

[11] Yu. I. Manin and Yu. Tschinkel, Points of bounded height on del Pezzo surfaces, Compositio Math. 85 (1993), 315-332.

[12] C. M. Skinner and T. D. Wooley, Sums of two kth powers, J. Reine Angew. Math. 462 (1995), 57-68.

[13] J. B. Slater and H. P. F. Swinnerton-Dyer, Counting points on cubic surfaces, I, Astérisque 251 (1998), 1-12.

Mathematical Institute

$24-29$ St. Giles'

Oxford OX1 3LB, U.K.

E-mail: browning@maths.ox.ac.uk

Received on 11.3.2002

and in revised form on 18.10.2002 